\title{
TENDENCIAS DE LA PRECIPITACIÓN EN EL NORTE GRANDE DE CHILE Y SU RELACIÓN CON LAS PROYECCIONES DE CAMBIO CLIMÁTICO
}

\author{
PRECIPITATION TRENDS IN THE CHILEAN NORTE GRANDE AND \\ ITS RELATIONSHIP WITH CLIMATE CHANGE PROJECTIONS
}

\author{
Pablo Sarricolea*, Óliver Meseguer Ruiz**, Hugo Romero Aravena****
}

\begin{abstract}
Se analizan las tendencias de la precipitación en el Norte Grande de Chile mediante el test no paramétrico de Mann-Kendall y el test de Sen, lo que permite detectar, para las sesenta estaciones meteorológicas disponibles, la posibilidad de cambios decadales de la precipitación con significancia estadística $(\mathrm{p}<0,1)$. Los resultados muestran que es el Altiplano la región donde se aprecia una reducción significativa de la precipitación anual entre 1972 y 2013. La zona de precordillera posee tendencias de incremento para Poroma y San Pedro de Atacama. El desierto costero no muestra tendencias significativas, lo que implica la mantención de las condiciones hiperáridas. Estos resultados son comparables con las proyecciones de cambio climático a fines del siglo XXI. Se concluye la importancia de seguir monitoreando las tendencias, aumentar la densidad de estaciones a la vez que se mantienen las ya existentes.
\end{abstract}

Palabras clave: Altiplano, Chile, Mann-Kendall, Norte Grande, precipitación.

The precipitation trends in the Norte Grande region in Chile are analysed using the Mann-Kendall non-parametric test and the Sen's trend test, to detect, for 60 available meteorological stations, possible statistically significant $(p<0.01)$ decadal precipitation changes. Results show that the Altiplano is the region where a significant reduction of annual precipitation between 1972 and 2013 could be observed. The Precordillera region records increase trends for Poroma and San Pedro de Atacama. No significant trends were found in the coastal desert region, where hyper arid conditions are maintained. Those results may be compared to the climate change projections to the end of the 21 st century. As a conclusion, it's important to keep trends monitoring, increase density of meteorological stations and maintain those already existing.

Key words: Altiplano, Chile, Mann-Kendall, Norte Grande, precipitation.

\section{Introducción}

El Norte Grande de Chile corresponde a una de las áreas más áridas del mundo, con mayor radiación solar y gradiente altitudinal (Sarricolea y Romero 2015). El clima es uno de los componentes ambientales más característicos y significativos de las regiones del Norte Grande de Chile. En el Desierto de Atacama casi nunca llueve, e incluso en las zonas costeras las estaciones meteorológicas situadas en Arica $\left(18^{\circ} \mathrm{S}\right)$, Iquique $\left(19^{\circ} \mathrm{S}\right) \mathrm{y}$ Antofagasta $\left(23^{\circ} \mathrm{S}\right)$ poseen registros anuales de precipitación menores a $3 \mathrm{~mm}$ (Romero y Kampf 2003). Sin embargo, en las cordilleras y altiplanos andinos las lluvias aumentan considerablemente, ofertando los recursos hídricos que se requieren para el desarrollo de las ciudades, yacimientos mineros y limitada producción agrícola y ganadera. Sin duda, se trata de un área de contrastados paisajes (desierto costero y Altiplano andino), lo que se puede expresar en precipitación menor a 10 milímetros anuales (invernal y de origen sobre el océano Pacífico), bajo los 2000 metros de altitud, y más de 300 milímetros anuales en el Altiplano (de verano y de origen oriental y amazónico). Durante el Holoceno, esta región planetaria no ha estado ajena a una intensa variabilidad climática, la que se puede resumir en períodos más húmedos y secos que el actual. Entre los 17500 y 9500 años antes del presente, diversos autores coinciden en condiciones ambientales más húmedas (Santoro et al. 2011), y luego un silencio arqueológico

\footnotetext{
* Universidad de Chile, Departamento de Geografía. Santiago, Chile. Universidad de Barcelona, Grupo de Climatología Barcelona, España. Correo electrónico: psarricolea@uchilefau.cl

** Universidad de Tarapacá, Departamento de Ciencias Históricas y Geográficas. Arica, Chile. Universidad de Barcelona, Grupo de Climatología. Barcelona, España. Correo electrónico: omeseguer@uta.cl

*** Universidad de Chile, Departamento de Geografía. Laboratorio de medio ambiente y territorio. Santiago, Chile. Correo electrónico: hromero@uchilefau.cl
} 
asociado a sequías, de 9500 a 4500 años antes del presente (Núñez et al. 2002). Trenberth (2011) señala que se han observado, a mayor escala temporal, cambios de clima desértico a semiárido y viceversa en latitudes subtropicales, y cambios en los regímenes de humedad en latitudes altas.

Estudiar la precipitación en el Norte Grande es un desafío, pues el Grupo Intergubernamental de Expertos sobre el Cambio Climático (IPCC 2013) lo clasifica como una región de alta incertidumbre. Ello se debe a que la variabilidad de la precipitación expresada en desviaciones típicas supera a los valores de tendencia central (medias anuales), hecho que queda reflejado en la mayoría de las estaciones meteorológicas del desierto. En dicho sentido, Schulz et al. (2011) señalan que cambios en la precipitación en el desierto costero han pasado inadvertidos, y pocos estudios han examinado algunos aspectos de su evolución en las últimas décadas (Houston 2006). No obstante, en el Altiplano andino las causas de dicha incertidumbre parecen tener más relación con los cambios en la circulación atmosférica y la deforestación amazónica.

Pese a la incertidumbre a la que se alude respecto de la precipitación en el Norte Grande (IPCC 2013), el estudio de sus tendencias históricas observadas proporciona potenciales indicadores para la modelación de los patrones futuros del cambio climático $y$, por tanto, ofrece una contribución importante al debate científico acerca de este. Así lo señalan Souvignet et al. (2012), destacando la importancia de los climas locales a este respecto.

Los cortos y escasos registros de estaciones meteorológicas de los que se dispone en el Norte Grande dificultan el establecimiento de tendencias claras durante el siglo XX, referidas a aumentos o disminuciones interanuales de las temperaturas y de las precipitaciones. Estas últimas reflejan una alta irregularidad con alzas y disminuciones asociadas a patrones de teleconexiones (la más influyente, el ENSO). No obstante, al decir de Sarricolea y Romero (2015), los modelos climáticos globales (GCM) y regionales (RCM) construidos bajo los escenarios de emisiones de gases de efecto invernadero y variabilidad natural, señalan tendencias de aumentos significativos de las temperaturas, y escasos niveles de confianza sobre las tendencias de las precipitaciones de fines del siglo XXI (Fuenzalida et al., 2007), pese a que en Minvielle y Garreaud (2011) se menciona un debilitamiento de los flujos desde la cuenca amazónica, y por tanto, una disminución de la precipitación altiplánica, lo que es consistente con los resultados publicados por el Quinto Informe de Evaluación (AR5) del IPCC (2013: 1264).

Estudios recientes efectuados en el Norte Grande, aplicando el peor escenario de emisiones del AR5 del IPCC (2013), señalan un incremento de las temperaturas entre $2{ }^{\circ} \mathrm{C}$ y $5{ }^{\circ} \mathrm{C}$, y una reducción de las precipitaciones de más de $30 \%$, a excepción de una franja desértica del interior, entre la costa y la precordillera (Sarricolea y Romero 2015).

Por lo anteriormente mencionado, la presente investigación tiene como objetivo establecer en qué medida las tendencias observadas de la precipitación en el Norte Grande se corresponden con las variaciones propuestas por el AR5 en su peor escenario (Sarricolea y Romero 2015) y en el informe de cambio climático del Ministerio de Medio Ambiente del Gobierno de Chile (Santibáñez et al. 2016). La hipótesis de trabajo señala que pocas estaciones poseen tendencias estadísticas significativas, y que ellas corresponden principalmente al Altiplano andino, así como lo encontrado en Bennett et al. (2016). Para ello el trabajo consistió en la recopilación de la información tanto de estaciones meteorológicas como respecto de su proyección futura, y la detección de sus tendencias de cambio. Los resultados obtenidos fueron contrastados con la literatura científica disponible.

\section{Metodología y datos utilizados}

El Norte Grande de Chile se ubica en los Andes Centrales, región que comprende a la zona andina de Perú, Bolivia, Argentina y Chile. Se sitúa entre las latitudes $\sim 18^{\circ} \mathrm{S}$ y $\sim 27^{\circ} \mathrm{S}$. Se caracteriza según Latorre et al. (2005) por la existencia de un desierto costero (0-900 metros de altitud), la Depresión Intermedia (900-2200 metros de altitud), la precordillera (22003500 metros de altitud) y las mesetas semiáridas sobre los $3500 \mathrm{~m}$ de altitud, que en su conjunto forman lo que se denomina "Puna" o "Altiplano" (Jaksic et al. 1997). Administrativamente, corresponde a las regiones XV de Arica y Parinacota, I de Tarapacá y II de Antofagasta (Figura 1).

Se utilizan para este estudio los datos pluviométricos de sesenta estaciones meteorológicas, pertenecientes, en su mayoría (54 estaciones), a la Dirección General de Aguas (DGA), y seis que corresponden a la Dirección Meteorológica de Chile (DMC) (Tabla 1). Los datos mensuales fueron obtenidos desde el repositorio del Centro de Ciencia del Clima y la Resiliencia ((CR)2) de la Universidad 


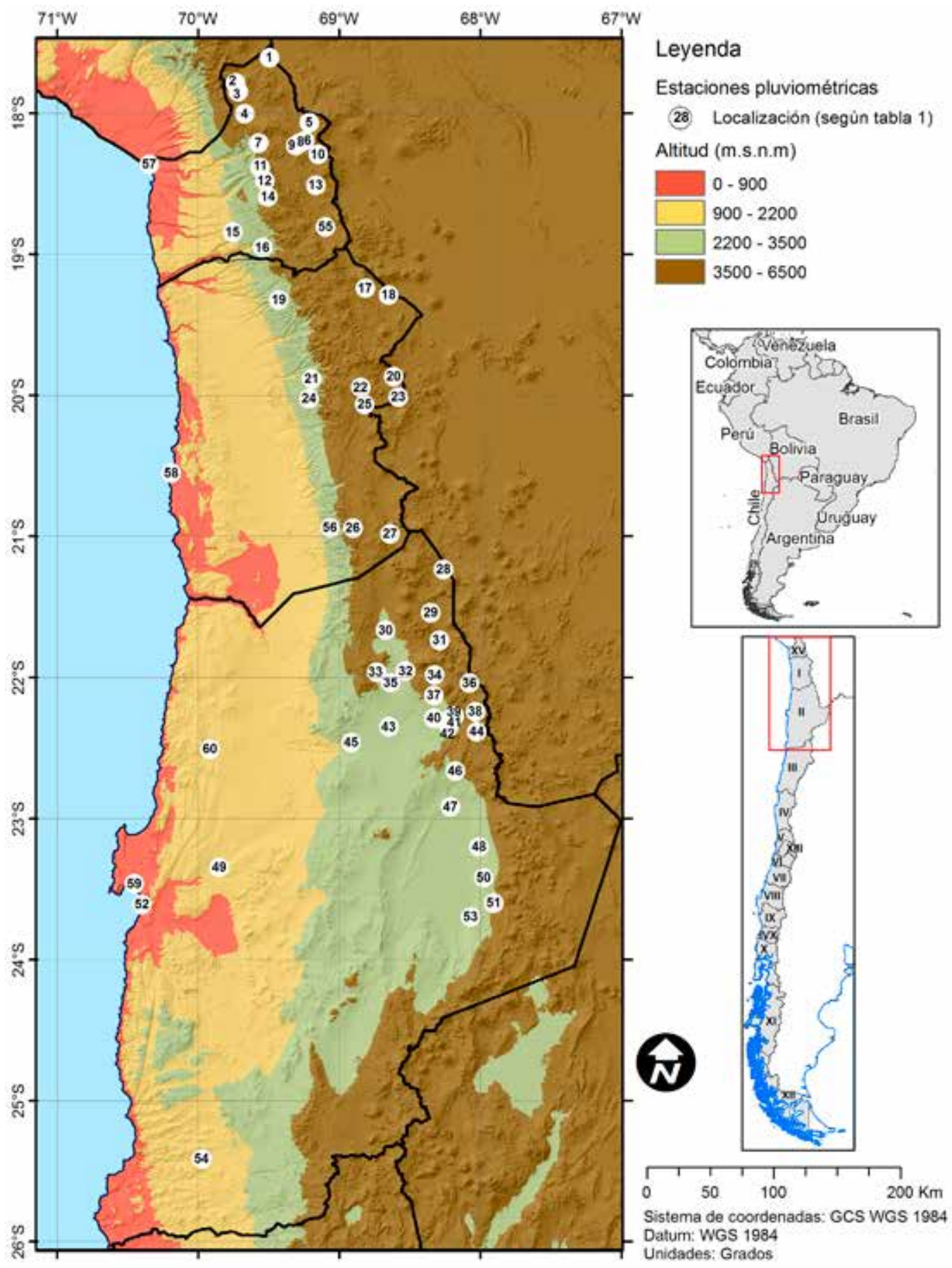

Figura 1: Área de estudio y estaciones meteorológicas utilizadas.

Fuente: Elaboración propia. 
Tabla 1: Estaciones meteorológicas utilizadas.

\begin{tabular}{|c|c|c|c|c|c|c|}
\hline ID & Nombre estación & Latitud $\left({ }^{\circ}\right)$ & Longitud $\left(^{\circ}\right)$ & Altitud (m) & Período & Número de años \\
\hline 1 & Visviri & $-17,60$ & $-69,48$ & 4080 & $1969-2015$ & 47 \\
\hline 2 & Villa Industrial (Tacora) & $-17,77$ & $-69,72$ & 4080 & $1975-2015$ & 41 \\
\hline 3 & Humapalca & $-17,84$ & $-69,70$ & 3980 & $1972-2015$ & 44 \\
\hline 4 & Alcérreca & $-17,99$ & $-69,66$ & 3990 & $1971-2015$ & 45 \\
\hline 5 & Caquena & $-18,05$ & $-69,20$ & 4400 & $1970-2015$ & 45 \\
\hline 6 & Cotacotani & $-18,18$ & $-69,23$ & 4550 & $1963-2008$ & 46 \\
\hline 7 & Putre & $-18,20$ & $-69,56$ & 3545 & $1978-2008$ & 31 \\
\hline 8 & Parinacota Conaf Dga & $-18,20$ & $-69,27$ & 4420 & $1933-2015$ & 74 \\
\hline 9 & Chucuyo Retén & $-18,22$ & $-69,30$ & 4400 & $1961-2015$ & 55 \\
\hline 10 & Chungara Retén & $-18,28$ & $-69,14$ & 4570 & $1963-2015$ & 50 \\
\hline 11 & Central Chapiquia & $-18,37$ & $-69,55$ & 3350 & $1963-2015$ & 53 \\
\hline 12 & Belén & $-18,47$ & $-69,52$ & 3240 & $1968-2015$ & 48 \\
\hline 13 & Guallatire & $-18,50$ & $-69,15$ & 4240 & $1969-2015$ & 47 \\
\hline 14 & Tignamar & $-18,58$ & $-69,49$ & 3230 & $1975-2015$ & 41 \\
\hline 15 & Codpa & $-18,83$ & $-69,74$ & 1870 & $1969-2015$ & 47 \\
\hline 16 & Esquia & $-18,94$ & $-69,53$ & 2170 & 1976-2014 & 39 \\
\hline 17 & Enquelca (Ex-Caraguano) & $-19,23$ & $-68,80$ & 3900 & $1985-2015$ & 27 \\
\hline 18 & Colchane (T. Isluga) & $-19,28$ & $-68,64$ & 3700 & 1976-2015 & 39 \\
\hline 19 & Camia & $-19,31$ & $-69,42$ & 2500 & $1971-2015$ & 42 \\
\hline 20 & Cancosa & $-19,86$ & $-68,60$ & 3930 & 1977-2011 & 34 \\
\hline 21 & Poroma & $-19,87$ & $-69,18$ & 2880 & $1975-2015$ & 41 \\
\hline 22 & Lagunillas (Pampa Lirima) & $-19,93$ & $-68,84$ & 4020 & $1983-2014$ & 31 \\
\hline 23 & Huaytani & $-20,00$ & $-68,57$ & 3950 & $1983-2015$ & 31 \\
\hline 24 & Parca & $-20,01$ & $-69,20$ & 2650 & $1978-2015$ & 38 \\
\hline 25 & Coyacagua & $-20,05$ & $-68,81$ & 4013 & $1962-2015$ & 54 \\
\hline 26 & Copaquire & $-20,93$ & $-68,89$ & 3540 & $1978-2010$ & 29 \\
\hline 27 & Ujina & $-20,97$ & $-68,63$ & 4300 & 1974-2015 & 36 \\
\hline 28 & Ollagüe & $-21,22$ & $-68,25$ & 3700 & $1972-2014$ & 31 \\
\hline 29 & Cebollar & $-21,53$ & $-68,34$ & 3730 & 1976-2006 & 27 \\
\hline 30 & Lequena & $-21,66$ & $-68,66$ & 3320 & 1984-2014 & 31 \\
\hline 31 & Ascotan & $-21,73$ & $-68,28$ & 3970 & $1975-2014$ & 37 \\
\hline 32 & Parshall N 2 & $-21,94$ & $-68,52$ & 3318 & 1970-2012 & 43 \\
\hline 33 & Conchi Viejo & $-21,95$ & $-68,72$ & 3491 & 1974-2014 & 38 \\
\hline 34 & Ojos San Pedro & $-21,97$ & $-68,31$ & 3800 & 1979-2014 & 36 \\
\hline 35 & Conchi Embalse & $-22,03$ & $-68,62$ & 3010 & 1976-2014 & 39 \\
\hline 36 & Inacaliri & $-22,03$ & $-68,07$ & 4040 & 1970-2014 & 45 \\
\hline 37 & Cupo & $-22,11$ & $-68,32$ & 3370 & 1979-2014 & 36 \\
\hline 38 & Linzor & $-22,23$ & $-68,02$ & 4100 & 1974-2014 & 41 \\
\hline 39 & Toconce & $-22,26$ & $-68,17$ & 3310 & $1973-2014$ & 42 \\
\hline 40 & Ayquina & $-22,28$ & $-68,32$ & 3031 & $1968-2014$ & 47 \\
\hline 41 & Salado Embalse & $-22,29$ & $-68,20$ & 3200 & $1976-2015$ & 40 \\
\hline 42 & Caspana & $-22,34$ & $-68,21$ & 3260 & 1972-2007 & 36 \\
\hline 43 & Chiu-Chiu & $-22,34$ & $-68,64$ & 2524 & $1975-2014$ & 40 \\
\hline 44 & El Tatio & $-22,37$ & $-68,01$ & 4370 & $1978-2015$ & 37 \\
\hline 45 & Calama & $-22,45$ & $-68,90$ & 2300 & $1967-2015$ & 46 \\
\hline 46 & Río Grande & $-22,65$ & $-68,17$ & 3250 & 1978-2014 & 37 \\
\hline 47 & San Pedro de Atacama & $-22,91$ & $-68,20$ & 2450 & 1961-1989 & 29 \\
\hline 48 & Toconao Experimental & $-23,19$ & $-68,00$ & 2500 & 1976-2007 & 32 \\
\hline 49 & Baquedano & $-23,33$ & $-69,84$ & 1032 & $1976-2015$ & 36 \\
\hline 50 & Camar & $-23,41$ & $-67,96$ & 2700 & 1980-2014 & 35 \\
\hline 51 & Socaire & $-23,59$ & $-67,89$ & 3251 & $1975-2014$ & 40 \\
\hline 52 & Antofagasta & $-23,60$ & $-70,39$ & 50 & 1979-2015 & 37 \\
\hline 53 & Peine & $-23,68$ & $-68,06$ & 2460 & 1975-2014 & 40 \\
\hline 54 & Aguas Verdes & $-25,40$ & $-69,96$ & 1560 & 1988-2014 & 27 \\
\hline 55 & Chilcaya Retén & $-18,79$ & $-69,08$ & 4279 & $1980-2008$ & 26 \\
\hline 56 & Huatacondo & $-20,93$ & $-69,05$ & 2299 & 1979-2006 & 28 \\
\hline 57 & Chacalluta Arica Ap. & $-18,35$ & $-70,34$ & 63 & $1958-2015$ & 58 \\
\hline 58 & Diego Aracena Iquique Ap. & $-20,54$ & $-70,18$ & 52 & $1981-2015$ & 35 \\
\hline 59 & Cerro Moreno Antofagasta Ap. & $-23,45$ & $-70,44$ & 113 & $1950-2015$ & 66 \\
\hline 60 & El Loa Calama Ad. & $-22,50$ & $-69,90$ & 2293 & $1967-2015$ & 48 \\
\hline
\end{tabular}

Fuente: Elaboración propia basada en DGA y DMC. 
de Chile, siendo revisados para evitar problemas relativos a la existencia de lagunas y respecto de su continuidad temporal. La longitud media de las series es de cuarenta años, cuyo período de registro de mayor continuidad abarca desde 1972 a 2013.

No se cuenta con una base de metadatos de las estaciones meteorológicas, por lo que, así como ocurre en el estudio de Bennett et al. (2016), se asume que todas las series son homogéneas. Para analizar las tendencias se aplicó el test de Mann-Kendall (Mann 1945; Kendall 1975), el que permite la detección de las tendencias estadísticamente significativas. Para estimar la magnitud de la tendencia se utilizó el método de la pendiente de Sen (Sen 1968). El test de Mann-Kendall es un test no paramétrico que evalúa el comportamiento monótono de una serie de datos, y es muy adecuado para el estudio de la precipitación, pues no requiere normalidad ni linealidad. En este artículo se consideran tendencias significativas aquellas que alcancen el primer nivel de confianza, $90 \%(\mathrm{p}<0,1)$. La pendiente de Sen es un método no paramétrico robusto que evalúa tendencias, con la ventaja de permitir la existencia de lagunas de datos.

Posteriormente se compararon los resultados de las tendencias observadas con el peor escenario, el RCP8.5 (Representative Concentration Pathways en inglés) del IPCC para el período 2061-2080 (Sarricolea y Romero 2015). Se obtuvieron simulaciones del clima del IPCC para el mismo período de análisis de la tendencia (1972-2013) y se analizó si las señales detectadas de las estaciones meteorológicas coinciden en alguna medida con el ensamble de simulaciones de IPCC. Para las simulaciones se recurrió al uso de Climate Explorer (www.climexp.knmi.nl) que es una aplicación para la investigación climática administrada por el Real Instituto Meteorológico de los Países Bajos (KNMI). Contiene una colección completa de conjuntos de datos climáticos y herramientas de análisis del CMIP5 (Coupled Model Intercomparison Project Phase 5). Se descargó el promedio de la precipitación en $\mathrm{mm} /$ día para 39 simulaciones, históricas y proyectadas del RCP8.5, correspondientes al cuadrante del Norte Grande $\left(17^{\circ} \mathrm{S}, 25^{\circ} \mathrm{S}, 68^{\circ} \mathrm{W}, 71^{\circ} \mathrm{W}\right)$.

\section{Resultados}

\section{Análisis de la precipitación y sus tendencias}

Como se ha señalado anteriormente, la precipitación anual media fluctúa considerablemente en el área de estudio, variando entre $0,59 \mathrm{~mm}$ en Iquique (costa) y 394,79 mm en Cotacotani (altiplano andino; Tabla 2). Destaca la alta correlación entre la cantidad de lluvias medias anuales y su distribución zonal y altitudinal, con valores de Pearson de $-0,71$ $y+0,71(p<0,01)$ en ambos casos. El Coeficiente de Variación y la altitud se relacionan mejor $(r=$ 0,87 ), diferenciando la costa (alta variabilidad) del Altiplano (moderada variabilidad), lo que se aprecia en la Figura 2.

Respecto de las tendencias, se observa que, de las sesenta estaciones meteorológicas analizadas, solo en trece resultan significativas al $90 \%$, y, de ellas, solo siete al 95\% (Tabla 2). La magnitud de los cambios señala tendencias a la reducción de las precipitaciones en once de estas trece estaciones meteorológicas, con valores entre $-44,0$ y $-7,95$ $\mathrm{mm}$ /década. Dos estaciones ubicadas en el área de estudio, Poroma y San Pedro de Atacama, presentan tendencias significativas al alza de las precipitaciones, con 9,15 y 7,18 mm/década, respectivamente.

Geográficamente, la Figura 3 muestra que estaciones meteorológicas costeras y localizadas en la Depresión intermedia no manifiestan ninguna tendencia de cambio, mientras que las de precordillera y Altiplano exhiben tendencias generales a la reducción de la precipitación, con la excepción de Parinacota, en el Altiplano de Arica. Considerando las tendencias significativas, en el Altiplano se registran descensos de la precipitación, y aumentos para la precordillera de Tarapacá y Antofagasta.

\section{Comparación con las proyecciones del AR5}

La aplicación del método de anomalías y la evaluación de los cambios en la variabilidad de las precipitaciones en el período 2061-2080 coincide con las proyecciones del último informe del IPCC (2013) que prevé considerables descensos de la precipitación en el Altiplano (Sarricolea y Romero 2015), sobre todo en el extremo norte y sur de las regiones de Arica-Parinacota y Tarapacá. La situación no difiere de lo señalado en la región de Antofagasta por Santibáñez et al. (2016) para mediados del siglo XXI. No obstante, al confrontar la serie de las estaciones con las simulaciones de todos los modelos RCP8.5 se aprecia que en el período de registro común no se advierten coincidencias, e incluso se observan comportamientos contradictorios, lo que implica una alta incertidumbre, incluso en el período histórico (Figura 4), para la interpretación 
Tabla 2: Estaciones meteorológicas analizadas. La tendencia aparece en $\mathrm{mm} /$ década.

\begin{tabular}{|c|c|c|c|c|c|c|c|c|}
\hline ID & Nombre estación & Período & Test Z & Q década & Sig. & $\mathrm{P}$ & Desv. P & $\mathrm{CV}$ \\
\hline 1 & Visviri & $1969-2015$ & $-1,52$ & $-28,13$ & no sig. & 277,68 & 142,60 & 0,51 \\
\hline 2 & Villa Industrial (Tacora) & $1975-2015$ & $-2,19$ & $-44,00$ & 95 & 283,17 & 164,76 & 0,58 \\
\hline 3 & Humapalca & $1972-2015$ & $-0,84$ & $-13,28$ & no sig. & 289,64 & 151,87 & 0,52 \\
\hline 4 & Alcérreca & $1971-2015$ & $-1,91$ & $-25,00$ & 90 & 204,87 & 102,29 & 0,50 \\
\hline 5 & Caquena & $1970-2015$ & 0,36 & 8,88 & no sig. & 369,19 & 183,15 & 0,50 \\
\hline 6 & Cotacotani & $1963-2008$ & 0,23 & 4,26 & no sig. & 394,79 & 141,64 & 0,36 \\
\hline 7 & Putre & $1978-2008$ & 0,61 & 15,38 & no sig. & 197,58 & 108,25 & 0,55 \\
\hline 8 & Parinacota Conaf Dga & $1933-2015$ & $-0,70$ & $-5,52$ & no sig. & 313,25 & 131,10 & 0,42 \\
\hline 9 & Chucuyo Retén & $1961-2015$ & 0,30 & 3,47 & no sig. & 314,42 & 139,12 & 0,44 \\
\hline 10 & Chungara Retén & $1963-2015$ & $-1,17$ & $-11,61$ & no sig. & 282,66 & 122,12 & 0,43 \\
\hline 11 & Central Chapiquia & $1963-2015$ & 1,07 & 9,22 & no sig. & 140,64 & 106,21 & 0,76 \\
\hline 12 & Belén & $1968-2015$ & $-0,89$ & $-7,50$ & no sig. & 147,87 & 114,48 & 0,77 \\
\hline 13 & Guallatire & 1969-2015 & $-1,93$ & $-25,63$ & 90 & 258,02 & 137,13 & 0,53 \\
\hline 14 & Tignamar & 1975-2015 & 1,09 & 11,10 & no sig. & 120,30 & 97,19 & 0,81 \\
\hline 15 & Codpa & $1969-2015$ & 0,54 & 0,77 & no sig. & 17,04 & 15,45 & 0,91 \\
\hline 16 & Esquia & 1976-2014 & 0,31 & 2,27 & no sig. & 42,76 & 39,56 & 0,93 \\
\hline 17 & Enquelca (Ex-Caraguano) & $1985-2015$ & $-0,71$ & $-11,94$ & no sig. & 113,18 & 69,35 & 0,61 \\
\hline 18 & Colchane (T. Isluga) & 1976-2015 & $-0,35$ & $-2,89$ & no sig. & 121,03 & 80,42 & 0,66 \\
\hline 19 & Camia & $1971-2015$ & 1,60 & 3,50 & no sig. & 34,31 & 47,56 & 1,39 \\
\hline 20 & Cancosa & $1977-2011$ & $-0,27$ & $-7,00$ & no sig. & 150,25 & 114,45 & 0,76 \\
\hline 21 & Poroma & 1975-2015 & 2,34 & 9,15 & 95 & 44,33 & 35,86 & 0,81 \\
\hline 22 & Lagunillas (Pampa Lirima) & 1983-2014 & $-0,02$ & $-0,18$ & no sig. & 132,27 & 71,61 & 0,54 \\
\hline 23 & Huaytani & $1983-2015$ & $-0,03$ & $-1,36$ & no sig. & 139,22 & 111,63 & 0,80 \\
\hline 24 & Parca & $1978-2015$ & 1,01 & 2,82 & no sig. & 28,12 & 31,42 & 1,12 \\
\hline 25 & Coyacagua & $1962-2015$ & 0,27 & 1,62 & no sig. & 135,28 & 79,02 & 0,58 \\
\hline 26 & Copaquire & $1978-2010$ & 0,73 & 5,73 & no sig. & 79,94 & 110,61 & 1,38 \\
\hline 27 & Ujina & 1974-2015 & $-1,54$ & $-18,85$ & no sig. & 153,63 & 104,58 & 0,68 \\
\hline 28 & Ollagüe & 1972-2014 & $-4,03$ & $-28,67$ & 99 & 62,13 & 59,22 & 0,95 \\
\hline 29 & Cebollar & 1976-2006 & 0,13 & 0,97 & no sig. & 55,30 & 38,63 & 0,70 \\
\hline 30 & Lequena & 1984-2014 & $-1,05$ & $-7,14$ & no sig. & 87,35 & 122,90 & 1,41 \\
\hline 31 & Ascotan & $1975-2014$ & $-2,43$ & $-12,52$ & 95 & 65,67 & 45,42 & 0,69 \\
\hline 32 & Parshall N 2 & 1970-2012 & 0,26 & 0,48 & no sig. & 29,65 & 25,50 & 0,86 \\
\hline 33 & Conchi Viejo & 1974-2014 & 0,04 & 0,06 & no sig. & 37,31 & 38,71 & 1,04 \\
\hline 34 & Ojos San Pedro & 1979-2014 & $-3,08$ & $-18,39$ & 97 & 53,78 & 42,42 & 0,79 \\
\hline 35 & Conchi Embalse & 1976-2014 & $-0,38$ & $-0,63$ & no sig. & 18,17 & 16,97 & 0,93 \\
\hline 36 & Inacaliri & $1970-2014$ & $-1,16$ & $-10,44$ & no sig. & 119,27 & 88,65 & 0,74 \\
\hline 37 & Cupo & 1979-2014 & 0,75 & 4,23 & no sig. & 70,00 & 74,16 & 1,06 \\
\hline 38 & Linzor & 1974-2014 & $-1,94$ & $-25,64$ & 90 & 151,41 & 108,27 & 0,72 \\
\hline 39 & Toconce & 1973-2014 & $-1,81$ & $-15,36$ & 90 & 94,10 & 77,30 & 0,82 \\
\hline 40 & Ayquina & 1968-2014 & $-0,68$ & $-1,79$ & no sig. & 38,54 & 42,65 & 1,11 \\
\hline 41 & Salado Embalse & 1976-2015 & $-0,97$ & $-6,03$ & no sig. & 66,76 & 68,41 & 1,02 \\
\hline 42 & Caspana & 1972-2007 & $-1,89$ & $-17,09$ & 90 & 85,00 & 82,85 & 0,97 \\
\hline 43 & Chiu-Chiu & 1975-2014 & $-0,60$ & $-0,26$ & no sig. & 5,27 & 5,42 & 1,03 \\
\hline 44 & El Tatio & $1978-2015$ & $-1,54$ & $-17,52$ & no sig. & 133,33 & 101,62 & 0,76 \\
\hline 45 & Calama & $1967-2015$ & 0,44 & 0,00 & no sig. & 4,05 & 4,79 & 1,18 \\
\hline 46 & Río Grande & 1978-2014 & $-0,59$ & $-3,70$ & no sig. & 68,63 & 57,17 & 0,83 \\
\hline 47 & San Pedro de Atacama & 1961-1989 & 1,65 & 7,18 & 90 & 28,00 & 27,89 & 1,00 \\
\hline 48 & Toconao Experimental & 1976-2007 & $-2,00$ & $-7,95$ & 95 & 32,78 & 31,09 & 0,95 \\
\hline 49 & Baquedano & 1976-2015 & $-0,03$ & 0,00 & no sig. & 1,83 & 3,86 & 2,11 \\
\hline 50 & Camar & $1980-2014$ & $-1,56$ & $-7,08$ & no sig. & 34,12 & 31,88 & 0,93 \\
\hline 51 & Socaire & 1975-2014 & $-2,03$ & $-9,34$ & 95 & 38,96 & 38,36 & 0,98 \\
\hline 52 & Antofagasta & 1979-2015 & $-0,70$ & 0,00 & no sig. & 4,76 & 9,38 & 1,97 \\
\hline 53 & Peine & 1975-2014 & $-1,14$ & $-2,38$ & no sig. & 19,56 & 18,82 & 0,96 \\
\hline 54 & Aguas Verdes & 1988-2014 & 1,36 & 0,00 & no sig. & 6,13 & 9,99 & 1,63 \\
\hline 55 & Chilcaya Retén & $1980-2008$ & $-0,62$ & $-27,75$ & no sig. & 263,87 & 137,42 & 0,52 \\
\hline 56 & Huatacondo & 1979-2006 & 0,67 & 1,23 & no sig. & 13,39 & 15,06 & 1,12 \\
\hline 57 & Chacalluta Arica Ap. & $1958-2015$ & 0,34 & 0,00 & no sig. & 1,63 & 2,89 & 1,78 \\
\hline 58 & Diego Aracena Iquique Ap. & $1981-2015$ & $-0,84$ & 0,00 & no sig. & 0,59 & 1,83 & 3,09 \\
\hline 59 & Cerro Moreno Antofagasta Ap. & $1950-2015$ & $-0,21$ & 0,00 & no sig. & 3,55 & 6,10 & 1,72 \\
\hline 60 & El Loa Calama Ad. & $1967-2015$ & 1,11 & 0,17 & no sig. & 6,19 & 9,43 & 1,52 \\
\hline
\end{tabular}

Fuente: Elaboración propia. 


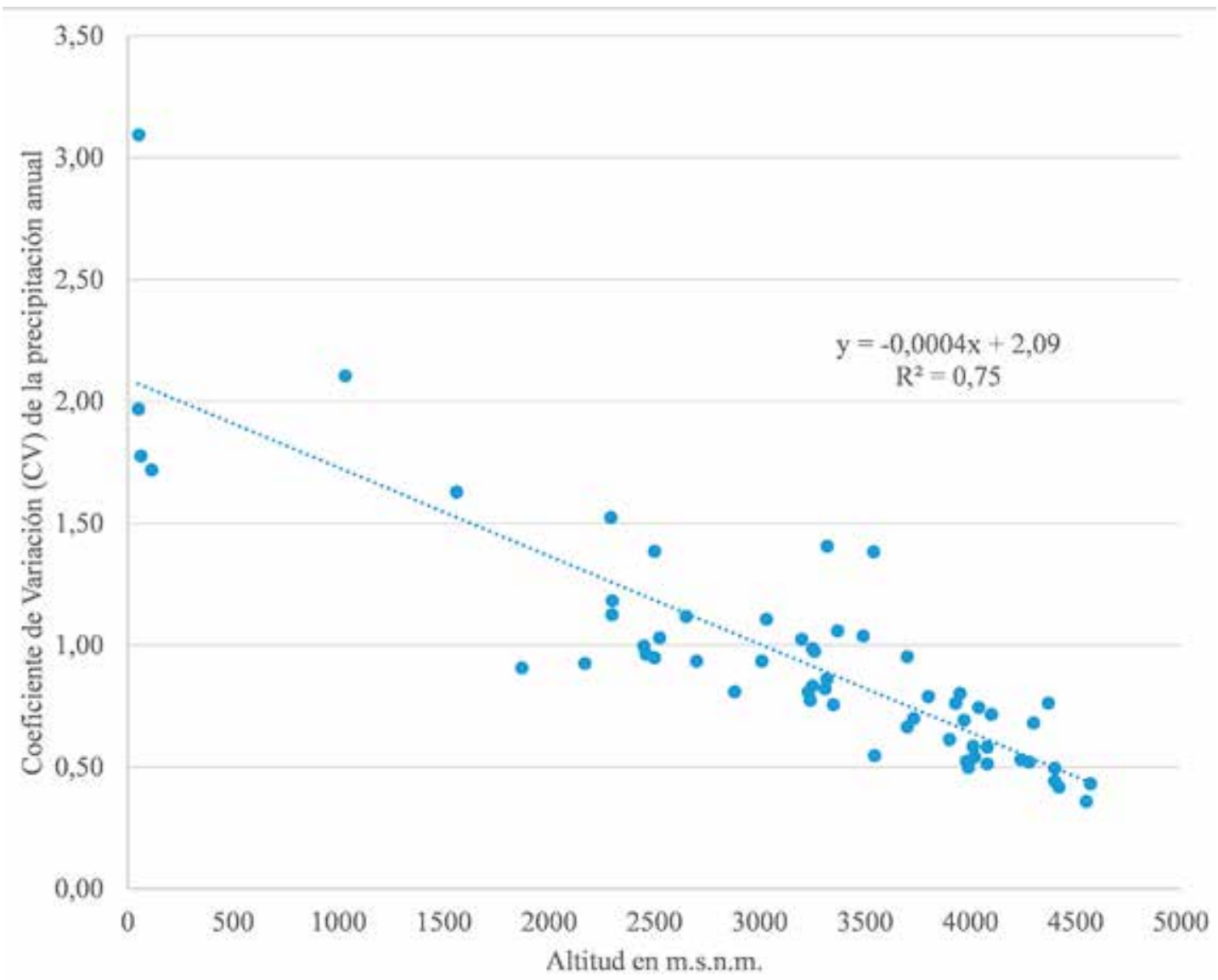

Figura 2: Correlación entre la altitud y el Coeficiente de Variación.

de los cambios en las precipitaciones de esta región chilena. Esto grafica las complicaciones de establecer relaciones entre las tendencias del clima regional y la actuación posible de forzantes radiactivos en las zonas áridas.

\section{Discusión y conclusiones}

Tratándose de paisajes complejos debido a las condiciones topográficas (altitud y exposición), en el Norte de Chile se manifiestan variabilidades espaciales y temporales de los climas, que caracterizan condiciones topoclimáticas que complementan o modifican las estimaciones de escala global, como lo anuncian las diferencias encontradas entre las zonas precordilleranas y altiplánicas. De mantenerse las tendencias de reducción de la precipitación encontradas en este estudio a escala regional, y señaladas por Bennett et al. (2016), es de esperar un aumento de la escasez de agua de origen pluvial y con ello la generación de problemas crecientes de disponibilidad del recurso para enfrentar las demandas, representadas en estas regiones, principalmente por la minería, urbanización y conservación de la naturaleza.

La región más afectada es y será el Altiplano andino (Sarricolea y Romero 2015), que constituye la única fuente de recarga moderna de las cuencas. De alcanzar las reducciones de precipitación, que aquí se advierten, se podrían materializar condiciones desérticas en estos paisajes a fines del siglo XXI.

La precordillera y parte de la Depresión Intermedia manifiestan, en dos estaciones, aumentos significativos de la precipitación. Ello es muy similar a lo proyectado por el AR5 (Sarricolea y Romero (2015).

Aún permanece la incertidumbre respecto de si se mantendrán las tendencias, e incluso cómo se comportarán otras fuentes de recursos hídricos, 


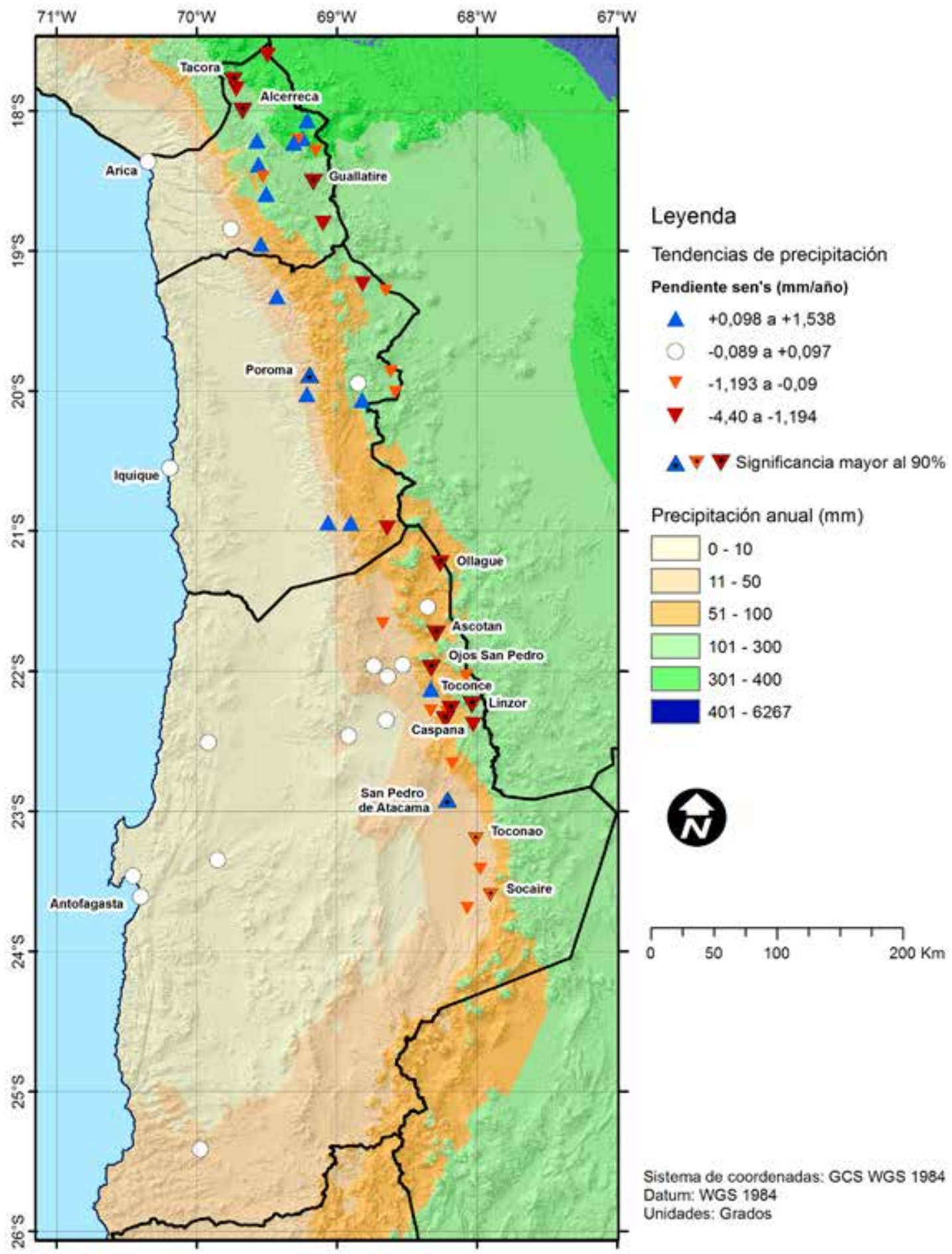

Figura 3: Mapa de tendencias de la precipitación del Norte Grande de Chile.

Fuente: Elaboración propia. 


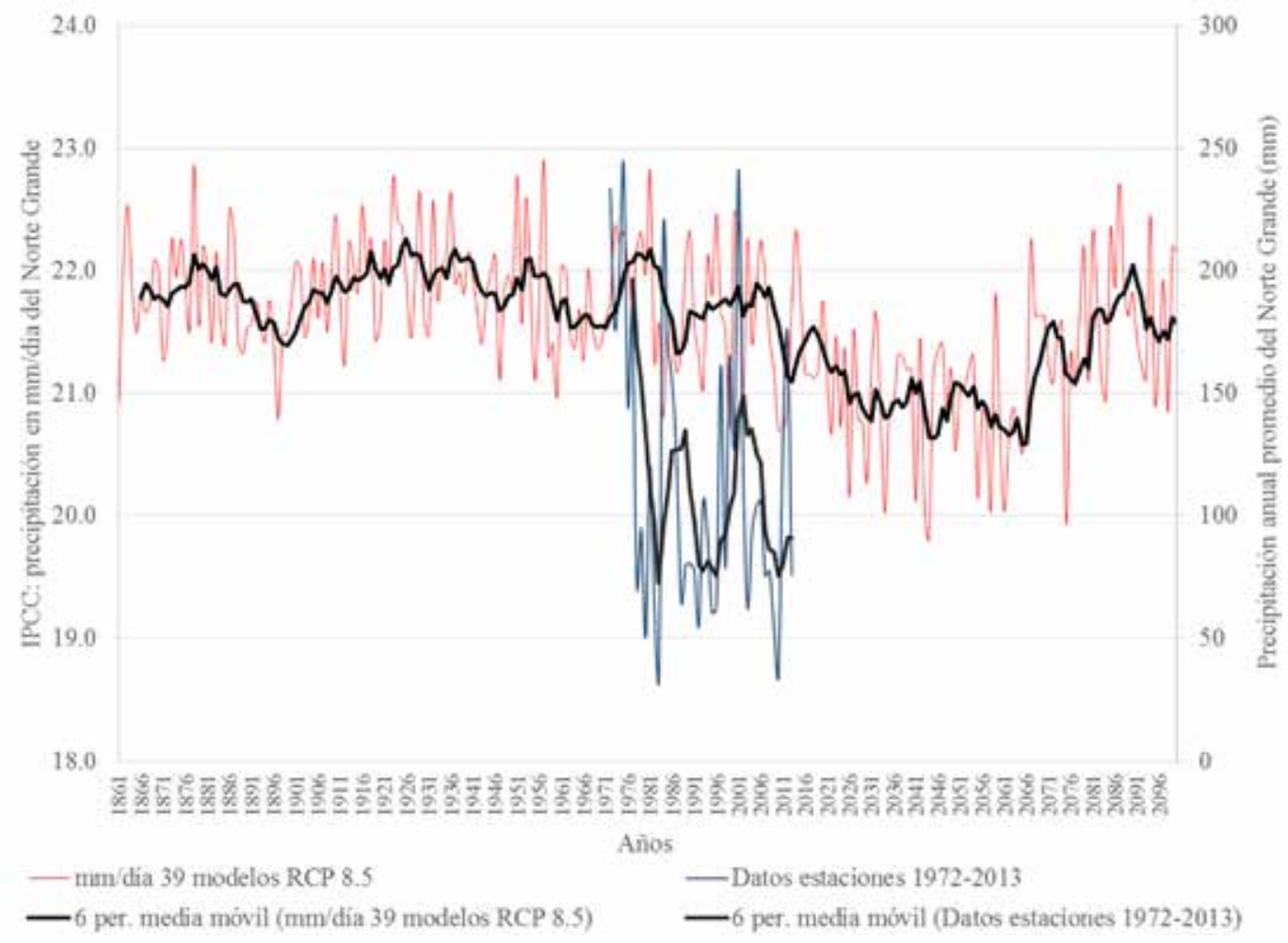

Figura 4: Precipitación histórica y proyectada (1861-2100) por el IPCC (CMIP5) para el área de estudio y el promedio observado por las estaciones meteorológicas usadas (1972-2013).

Fuente: Elaboración propia.

como las camanchacas o neblinas costeras. En este sentido, se debe considerar que este trabajo comparó cómo las tendencias en la precipitación se asemejan a alguno de los más de treinta modelos proyectados de cambio climático, lo que constituye un ejercicio metodológico que ayuda a entender la existencia de trayectorias observadas en las tendencias, y que se podrían agudizar en el futuro. No obstante, la incertidumbre es tan grande que no es posible asegurar que los cambios proyectados, más aún a fines de siglo XXI, sean totalmente atribuibles al cambio climático, y diferenciar la fracción que puede ser parte de la variabilidad natural del sistema. Las proyecciones del IPCC son ensambles que solo sugieren probabilidades de la dirección o trayectoria de cambio (Curry y Webster, 2011), pero en general no consideran comportamientos locales como los registrados por estaciones meteorológicas ubicadas en sitios de topografía compleja. De cualquier modo y debido al predominio de la variabilidad temporal y espacial de los climas en esta sección de Chile, es recomendable adoptar medidas precautorias y gestionar los ambientes y territorios en términos de incrementar sus niveles de resiliencia y capacidad adaptativa.

Es necesario mantener y aumentar la red de estaciones meteorológicas en el Norte Grande, y con ello mejorar las posibilidades de observar tendencias y ciclos que permitan mejorar las proyecciones de cambio climático.

Agradecimientos: Los autores quieren agradecer a la Dirección Meteorológica de Chile (DMC) y a la Dirección General de Aguas (DGA) por la cesión de los datos. También el apoyo institucional del Convenio de Desempeño UTA-MINEDUC, del proyecto UTA-Mayor 5744-16, de los proyectos FONDECYT 11130629, 1150701 y 11160059 , y del Grupo de Climatología (2014SGR300, Generalitat de Catalunya). Asimismo, también se agradece a los evaluadores del presente artículo por sus observaciones. 


\section{Referencias Citadas}

Bennett, M., New, M., Marino, J. \& Sillero-Zubiri C.

2016 Climate complexity in the Central Andes: A study case on empirically-based local variations in the dry Puna. Journal of Arid Environments 128:40-49.

Curry, J. A., \& Webster, P. J.

2011 Climate science and the uncertainty monster. Bulletin of the American Meteorological Society, 92(12):1667-1682.

Fuenzalida, H., Aceituno, P., Falvey, M., Garreuad, R.D., Rojas,

M. \& Sánchez, P.

2007 Study on climate variability for Chile during the 21 st century. Technical Report prepared for the National Environmental Committee. Santiago de Chile.

Houston, J.

2006 Variability of precipitation in the Atacama Desert: its causes and hydrological impact. International Journal of Climatology, 26(15): 2181-2198.

IPCC

2013 Climatic Change 2013: The Physical Science Basis. Working Group I Contribution to the Fifth Assessment Report of the Intergovernmental Panel on Climate Change. Cambridge University Press, New York.

Jaksic, F. M., Marquet, P. A., \& González, H.

1997 Una perspectiva ecológica sobre el uso del agua en el Norte Grande. Estudios públicos, 68: 171-195.

Kendall, M.G.

1975 Rank correlation methods. London: Charles Griffin.

Latorre, C., Betancourt, J. L., Rech, J. A., Quade, J., Holmgren,

C., Placzek, C., Maldonado, A., Vuille, M. \& Rylander, K. 2005 Late Quaternary history of the Atacama Desert. 23 S: the archaeology and environmental history of the southern deserts, 73-90.

Mann, H.B.

1945 Nonparametric tests against trend. Econometrica 13:245-259.

Minvielle, M., \& Garreaud, R. D.

2011 Projecting rainfall changes over the South American Altiplano. Journal of Climate, 24(17): 4577-4583.
Núñez, L., Grosjean, M., \& Cartajena, I.

2002 Human occupations and climate change in the Puna de Atacama, Chile. Science, 298(5594): 821-824.

Romero, H., \& Kampf, S.

2003 Impacts of Climate Fluctuations and Climate Changes on the Sustainable Development of the Arid Norte Grande in Chile. In Climate and Water, 83-115. Springer Netherlands.

Santibáñez, F., Santibáñez, P. \& González, P.

2016 Elaboración de una base digital del clima comunal de Chile: línea base (1980-2010) y proyección al año 2050. Estudio encargado por el Ministerio del Medio Ambiente Departamento de Cambio Climático, Chile.

Santoro, C. M., Osorio, D., Standen, V. G., Ugalde, P. C., Herrera,

K., Gayó, E. M., Rothhammer, F. \& Latorre, C.,

2011 Ocupaciones humanas tempranas y condiciones paleoambientales en el Desierto de Atacama durante la transición Pleistoceno-Holoceno. Boletín de arqueología PUCP, 15: 295-314.

Sarricolea, P., \& Romero, $\mathrm{H}$.

2015 Variabilidad y cambios climáticos observados y esperados en el Altiplano del norte de Chile. Revista de geografía Norte Grande, 62: 169-183.

Sen, P.K.

1968 Estimates of the regression coefficient based on Kendall's tau. Journal of the American Statistical Association, 63:1379-1389.

Schulz, N., Bosier, J.P. \& Aceituno, P.

2011 Climate change along the arid coast of northern Chile. International Journal of Climatology 32(12):1803-1814.

Souvignet, M., Oyarzún, R., Verbist, K. M., Gaese, H., \& Heinrich, J.

2012 Hydro-meteorological trends in semi-arid north-central Chile (29-32 S): water resources implications for a fragile Andean region. Hydrological Sciences Journal, 57(3): 479-495.

Trenberth, K. E.

2011 Changes in precipitation with climate change. Climate Research, 47(1-2): 123-138. 